\title{
Quantized Vortex Rings and Loop Solitons
}

\author{
P. J. Green ${ }^{1}$ · M. J. Grant ${ }^{1}$ · J. W. Nevin ${ }^{1}$ ·P. M. Walmsley ${ }^{1}$ A. I. Golov ${ }^{1}$ (D)
}

Received: 15 July 2019 / Accepted: 8 August 2020 / Published online: 29 August 2020

(c) The Author(s) 2020

\begin{abstract}
The vortex filament model is used to investigate the interaction of a quantized vortex ring with a straight vortex line and also the interaction of two solitons traveling in opposite directions along a vortex. When a ring reconnects with a line, we find that a likely outcome is the formation of a loop soliton. When they collide, loop solitons reconnect as they overlap each other producing either one or two vortex rings. These simulations are relevant for experiments on quantum turbulence in the zero temperature limit where small vortex rings are expected to be numerous. It seems that loop solitons might also commonly occur on vortex lines as they act as transient states between the absorption of a vortex ring before another ring is emitted when the soliton is involved in a reconnection.
\end{abstract}

Keywords Quantized vortices · Solitons · Vortex rings · Quantum turbulence

\section{Introduction}

The dynamics of quantized vortices in the limit of zero temperature are interesting due to the broad range of length scales and the rich variety of physics involved $[1,2]$. The dissipation of turbulent vortex tangles is thought to be due to the emission of phonons from short-wavelength Kelvin waves and also the release of small vortex rings that can travel to the walls of the container unimpeded. The vast majority of theoretical and computational research on quantum turbulence has focused only on these two types of vortex structure: linear helical deformations (Kelvin waves) [3-5] and vortex rings [6, 7]. However, other types of nonlinear deformations are possible (e.g., such as breathers [8]), and in this paper, we investigate whether one particular type of nonlinear deformation, loop solitons

P. M. Walmsley

paul.walmsley@manchester.ac.uk

A. I. Golov

andrei.golov@manchester.ac.uk

1 Department of Physics and Astronomy, The University of Manchester, Manchester M13 9PL, United Kingdom 
[9], may have a role to play in the dynamics of quantum turbulence. Solitons are solitary waves that can travel large distances without any change of shape or dissipation and can be found in a wide range of physical systems including fluids, optics, atomic and condensed matter physics [10].

In this work, we start by investigating the interaction between a vortex ring and a straight vortex line. Previous research has only considered the special case where the ring is initially travelling perpendicular to the line [11-15]. It was found that when the ring reconnects with the line it is either absorbed, with the energy transferred to packets of Kelvin waves, or a smaller secondary ring is emitted. In this work, we consider a more general case where the initial angle between the direction of the ring's motion and the line is varied. Our motivation is that as new experiments are currently being developed to allow visualization of vortices in the zero temperature limit $[16,17]$, then it is important to gain a more detailed understanding of some of the processes that could occur deep within the quantum regime of quantum turbulence (e.g., on length scales smaller than the typical intervortex spacing).

\section{Ring-Line Interactions}

The simulations in this paper used the vortex filament method, where quantized vortex lines are treated as infinitesimally thin space curves that are represented by a series of discrete mesh points. The velocity along any part of the vortex is given by the Biot-Savart law. The details of how this method is implemented can be found elsewhere $[5,18]$ (reconnections were performed using the Type-III method described by Baggaley [19]). For all the simulations in this paper, we use values for all parameters corresponding to superfluid ${ }^{4} \mathrm{He}$ in the zero temperature limit.

We investigate the interaction between a vortex ring of initial radius $R_{0}=0.5 \mu \mathrm{m}$ with a straight vortex line oriented along the $z$-axis with anticlockwise circulation. Periodic boundary conditions are applied at $z= \pm 10 \mu \mathrm{m}$. The ring is initially centered at $\left(-5 R_{0}, b, 0\right)$ where $b$ is a variable impact parameter and the direction of the velocity of the ring is at an angle $\theta$ with respect to the $x y$ plane. This initial configuration is shown in Fig. 1i. The spacing between vortex mesh points was $\delta \simeq 80 \mathrm{~nm}$ and the time step was set such that 25 steps corresponded to the period of a Kelvin wave with the shortest possible wavelength [5]. The simulations were allowed to proceed until a time of $0.5 \mathrm{~ms}$ had elapsed before the final state was characterized.

For sufficiently small values of $b(\theta)$, the ring reconnects with the line. There were three types of outcome following a reconnection-(i) the absorption of the ring and generation in certain cases of stable hump solitons, (ii) the creation of a stable loop soliton and (iii) the emission of a secondary vortex ring following a subsequent self-reconnection; each was accompanied by creation of Kelvin waves of a range of wavelengths. An example snapshot is shown in Fig. 1i which shows the creation of a loop soliton which spirals upward along the line.

Hasimoto showed that there is a family of possible solitons [9] on a straight vortex line with spatial dependence, 


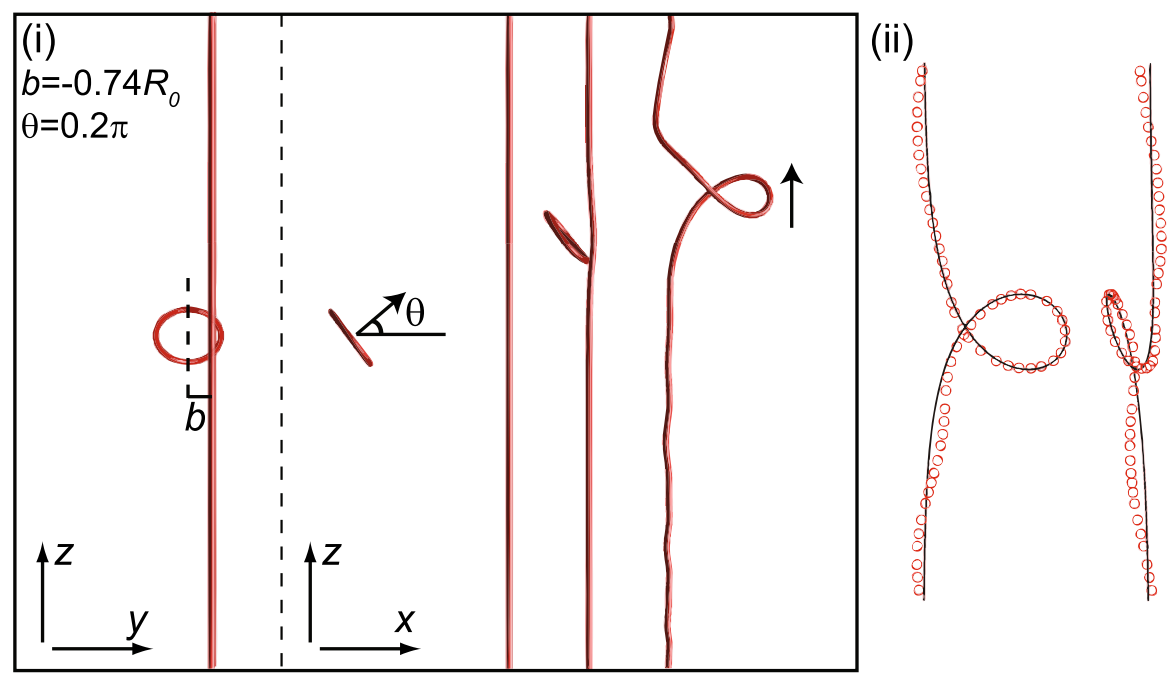

Fig. 1 i Snapshots showing the initial configuration (on the left, in two orthogonal projections) and then two stages of the formation of a loop soliton after the ring has reconnected with the line (on the right, projected on $(x, z)$ plane). ii Comparison of a loop soliton created via a ring reconnection and the Hasimoto solution (solid line, Eqs. 1-3) with parameters extracted from the velocity and angular velocity of the soliton (red circles indicated the vortex mesh points). The left and right plots show the $y z$ and $x z$, projections, respectively (Color figure online)

$$
\begin{gathered}
x_{\mathrm{sol}}=r_{\text {max }} \operatorname{sech}(v s) \cos \phi, \\
y_{\mathrm{sol}}=r_{\text {max }} \operatorname{sech}(v s) \sin \phi, \\
z_{\mathrm{sol}}=s-r_{\max } \tanh (v s)+z_{0},
\end{gathered}
$$

where $s$ is an arc length along the vortex filament and $z_{0}$ is the position of the centre of the soliton on the line, $\phi=T v s+\phi_{0}$ is an azimuthal angle where $\phi_{0}$ is a constant and $r_{\max }=2 / v\left(1+T^{2}\right)$ is the amplitude of the soliton. There are two parameters $T$ and $v$ that fully characterize the shape and size of the soliton: $v$ is a scale parameter that is equal to half of the maximum curvature and $T$ is a dimensionless parameter that controls the shape of the soliton. For $T>1$, the soliton is a helical wavepacket known as a hump soliton but for $0<T<1$ the soliton is a loop soliton ( $x_{\text {sol }}$ and $y_{\text {sol }}$ are multivalued functions of $z$, as can be seen in Fig. 1ii). Hasimoto solitons are solutions in the limit of the local induction approximation where only the local curvature contributes to the velocity. When non-local contributions are included, then the solitons weakly radiate Kelvin waves [20] but for the timescales considered in these simulations these dissipative losses can be neglected.

When a soliton was created following a ring-line reconnection, the time dependences of $z_{0}$ and $\phi$ were tracked and both were found to increase linearly with time, allowing the velocity $v$ and angular velocity $\omega$ of the soliton to be extracted. The Hasimoto solutions have 


$$
\begin{gathered}
\nu=\frac{v T \kappa}{2 \pi} \Lambda, \\
\omega=\frac{\nu^{2} \kappa}{4 \pi}\left(1+T^{2}\right) \Lambda,
\end{gathered}
$$

where $\kappa$ is the quantum of circulation, $a_{0}=0.13 \mathrm{~nm}$ is the vortex core parameter and $\Lambda \approx \ln \left(2 / \nu T a_{0}\right)$ which we checked using simulations of individual solitons with the range of $T$ and $v$ values found in these simulations. We were thus able to use Eqs. 4 and 5 to extract values for $T$ and $v$. These could be used to calculate $r_{\max }$ which gave values very close to what was seen in the simulation. Figure 1ii shows a comparison of a loop soliton created after a ring-line reconnection with the Hasimoto solution using the extracted values $T$ and $v$ which shows excellent agreement.

The simulations were repeated for a range of $b$ and $\theta$ values. The outcome is summarized in Fig. 2. The range of $b$ that results in a reconnection without re-emitting a secondary ring increases as $\theta \rightarrow \pi / 2$ due to non-local effects having a greater time to act due to the reduced closing velocity between the ring and the line (although reconnections are not possible for $\theta=\pi / 2$ ). Figure 2 also shows that the creation of a loop soliton always occurs following a reconnection if $\theta \gtrsim \pi / 4$, whereas the emission of a secondary ring or the absorption of the ring (leading to the generation of Kelvin waves and hump solitons) dominate as $\theta \rightarrow 0$. Solitons with very low values of $T<0.1$ are not stable in the full Biot-Savart model and a self-reconnection leads to the emission of a vortex ring. The precise conditions required for the conversion of the ring into the soliton on the line would involve considering the conservation of linear and angular impulse which is beyond the scope of this paper and a detailed analysis will be published

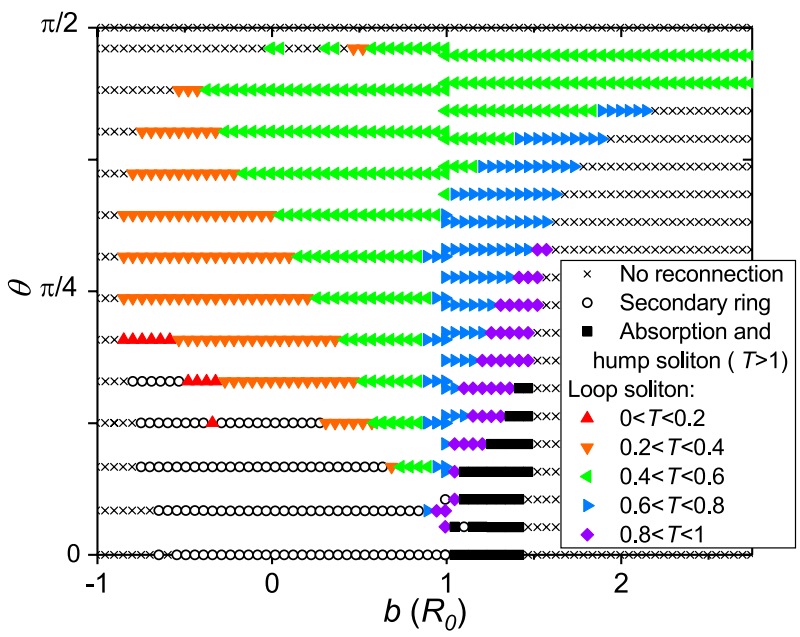

Fig. 2 Phase diagram indicating the outcome for the interaction between a straight vortex line and a vortex ring with initial impact parameter $b$ and angle $\theta$ (Color figure online) 
elsewhere. The important point is that loop solitons were found to be the most probable outcome following a reconnection of a vortex ring with a vortex line.

\section{Soliton-Soliton Interactions}

We have also investigated whether there is any interaction between two identical solitons moving in opposite directions along an otherwise straight vortex line with initial minimum separation of $3 \mu \mathrm{m}$. Eqs. 2-4 were used to initialize the solitons with the lower soliton having a variable initial phase offset, $0 \leq \phi_{0} \leq \pi$, relative to the upper soliton. Both solitons had $v=2 \mu \mathrm{m}^{-1}$ and the same magnitude of $T$ (i.e., the same shape and amplitude) but the upper soliton had a negative value of $T$ so that it moved down the line (negative $z$-direction).

Two examples from these simulations are shown in Fig. 3. A few different outcomes are observed. For high $T$ values the solitons will pass through each other with no reconnection taking place before continuing to move away from each other with their shapes unchanged. It is well known that hump solitons tend to pass through each other without changing shape [21-23] (although there can be a phase shift). However, the interaction between loop solitons generally leads to the solitons reconnecting with each other with the emission of one or two vortex rings, with some Kelvin wave deformations left behind on the line. Figure 4 summarizes the outcome of these simulations as a function of $T$ and $\phi_{0}$. We have also investigated varying the relative initial amplitude of the solitons and found that the maximum value of the shape parameter that leads to reconnections is $T_{\max } \approx 1.2 / A_{0}$ where $A_{0}$ is the ratio of the initial soliton amplitudes. This relationship was found to hold up to the highest value of $A_{0}=3$ that was used. It seems that as the two solitons become increasingly dissimilar in amplitude then the range of $T$ values producing a reconnection becomes smaller, such that only the more strongly looped solitons (i.e., low $T$ ) reconnect.

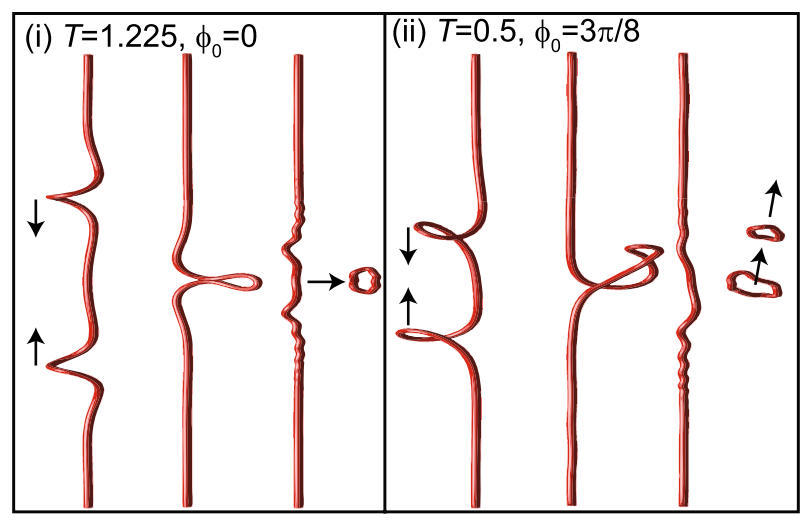

Fig. 3 Examples of the interaction between two identical solitons moving in opposite directions: i reconnection leading the emission of one vortex ring and ii two vortex rings 


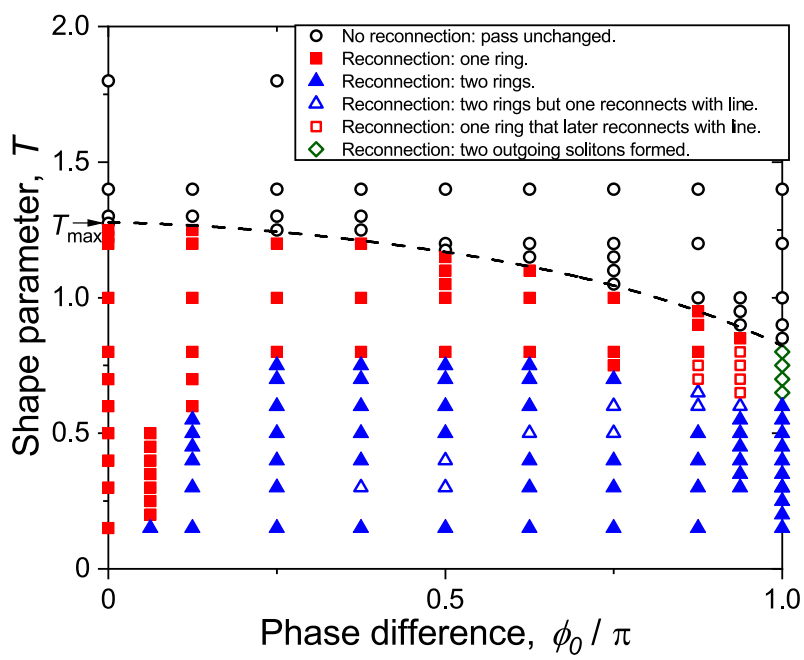

Fig. 4 Phase diagram indicating the outcome of the interaction between two identical solitons with shape parameter $T$ and an initial phase difference $\phi_{0}$. No reconnection occurs above the dashed line and the solitons pass each other. The green open diamonds indicate special cases for $\phi_{0}=\pi$ when there are two reconnections between the incoming solitons at the same time but no vortex rings are produced, instead, two different solitons form that subsequently move away from each other. The red open squares indicate cases where the solitons reconnect and a vortex ring is emitted but this vortex ring later reconnects back with the line, forming another soliton (Color figure online)

\section{Summary}

These simulations show that the creation of a loop soliton is a likely outcome when a ring reconnects with a line. In the absence of other deformations these loop solitons are stable and can travel large distances $\left(\gg R_{\max }\right)$ with minimal dissipative losses. However, the interaction with another soliton (and potentially other types of deformation) can lead to the emission of at least one vortex ring. This process could be repeated several times until the ring reaches the wall of the container. Our main finding is that solitons may play an important role in maintaining the identity of vortex rings rather than them simply just being reabsorbed within a tangle [24] with the line length being transferred to Kelvin waves. We suggest that a turbulent vortex tangle in the zero temperature limit can be thought of as a gas of solitons [23] and Kelvin waves on vortex lines interacting with a gas of vortex rings and phonons in the bulk-with frequent interconversion of rings to solitons and vice versa. Such features may eventually be observable using the visualization techniques currently being developed for the zero temperature limit or through experiments using charged ions trapped on vortices [25].

Acknowledgements The authors thank Dr. H. Salman for useful discussions. This work was funded by the Engineering and Physical Sciences Research Council (Grant No. EP/P025625/1). 
Open Access This article is licensed under a Creative Commons Attribution 4.0 International License, which permits use, sharing, adaptation, distribution and reproduction in any medium or format, as long as you give appropriate credit to the original author(s) and the source, provide a link to the Creative Commons licence, and indicate if changes were made. The images or other third party material in this article are included in the article's Creative Commons licence, unless indicated otherwise in a credit line to the material. If material is not included in the article's Creative Commons licence and your intended use is not permitted by statutory regulation or exceeds the permitted use, you will need to obtain permission directly from the copyright holder. To view a copy of this licence, visit http://creativecommons.org/licen ses/by/4.0/.

\section{References}

1. C.F. Barenghi, L. Skrbek, K.R. Sreenivasan, Proc. Natl. Acad. Sci. 111, 4647 (2014)

2. P. Walmsley, D. Zmeev, F. Pakpour, A. Golov, Proc. Natl. Acad. Sci. 111, 4691 (2014)

3. A.W. Baggaley, J. Laurie, Phys. Rev. B 89, 014504 (2014)

4. L. Kondaurova, V. L'vov, A. Pomyalov, I. Procaccia, Phys. Rev. B 90, 094501 (2014)

5. A.W. Baggaley, C.F. Barenghi, Phys. Rev. B 83, 134509 (2011)

6. M. Kursa, K. Bajer, T. Lipniacki, Phys. Rev. B 83, 014515 (2011)

7. R.M. Kerr, Phys. Rev. Lett. 106, 224501 (2011)

8. H. Salman, Phys. Rev. Lett. 111, 165301 (2013)

9. H. Hasimoto, J. Fluid. Mech. 51, 477 (1972)

10. T. Dauxois, M. Peyrard, Physics of Solitons (Cambridge University Press, Cambridge, England, 2006)

11. K.W. Schwarz, Phys. Rev. 165, 323 (1968)

12. K.W. Schwarz, Phys. Rev. B 31, 5782 (1985)

13. M. Tsubota, S. Maekawa, J. Phys. Soc. Jpn. 61, 2007 (1992)

14. J. Laurie, A.W. Baggaley, J. Low Temp. Phys. 180, 95 (2015)

15. A. Villois, H. Salman, D. Proment, J. Low Temp. Phys. 180, 68 (2015)

16. D.E. Zmeev et al., Phys. Rev. Lett. 110, 175303 (2013)

17. S.L. Ahlstrom et al., J. Low Temp. Phys. 175, 725 (2014)

18. T. Zhu et al., Phys. Rev. Fluids 1, 044502 (2016)

19. A.W. Baggaley, J. Low Temp. Phys. 168, 18 (2012)

20. S. A. Strong and L. D. Carr, arXiv:1712.05885 (2017)

21. K. Konno, M. Mituhashi, Y.H. Ichikawa, Chaos Solitons Fractals 1, 55 (1991)

22. K. Konno, Y.H. Ichikawa, Chaos Solitons Fractals 2, 237 (1992)

23. I. Redor et al., Phys. Rev. Lett. 122, 214502 (2019)

24. E.V. Kozik, B.V. Svistunov, J. Low Temp. Phys. 156, 215 (2009)

25. A.I. Golov, P.M. Walmsley, P.A. Tompsett, J. Low Temp. Phys. 161, 509 (2010)

Publisher's Note Springer Nature remains neutral with regard to jurisdictional claims in published maps and institutional affiliations. 